\title{
Anxiety and depression in Parkinson's disease
}

\author{
S.E. Starkstein ${ }^{1,2,3}$, R.G. Robinson ${ }^{1,2}$, R. Leiguarda ${ }^{3}$ and T.J. Preziosi ${ }^{4}$ \\ Departments of ${ }^{1}$ Psychiatry and Behavioral Sciences and ${ }^{4}$ Neurology, Johns Hopkins \\ University School of Medicine, Baltimore, MD 21205, ${ }^{2}$ Department of Psychiatry, \\ University of lowa, lowa City, IA 52242, USA and ${ }^{3}$ Raúl Carrea Institute of Neurological \\ Research, Buenos Aires, Argentina
}

Correspondence to: S.E. Starkstein, Instituto de Investigaciones Neurológicas Raúl Carrea, Ayacucho 2166, 1112 Buenos Aires, Argentina

\begin{abstract}
We examined a consecutive series of 40 patients with Parkinson's disease (PD) for the presence of anxiety. We found that $40 \%$ met DSM-III criteria for generalized anxiety disorders, and half of them also met criteria for either major depression or minor depression. While depression was associated with long duration of illness and more severe cognitive and physical impairments, anxiety was not associated with greater impairment. We conclude that anxiety, with or without depression, constitutes a frequent psychiatric problem among patients with PD.
\end{abstract}

Keywords: Anxiety - Depression - Parkinson's disease

\section{INTRODUCTION}

Psychiatric disorders are well-known findings among patients with Parkinson's disease (PD) (Cummings, 1992). Depression, for instance, has been reported in $50 \%$ of patients with PD (Cummings, 1992). In cross-sectional studies major depression was significantly correlated with duration of illness, severity of impairments, and cognitive deficits (Mayeux et al., 1981; Starkstein et al., 1989, 1990b). Recent longitudinal prospective studies demonstrated that the presence of depression significantly predicted a more rapid cognitive and physical decline (Starkstein et al., 1990a, 1992).

While depression in PD has been the focus of intense research, scant attention has been given to anxiety disorders. Schiffer et al. (1988) examined 16 depressed patients with PD and 20 depressed patients with multiple sclerosis, and found that PD patients had a significantly higher frequency of generalized anxiety disorders (GAD) and panic attacks. In a study that included 24 patients with PD, Stein et al. (1990) found that $38 \%$ of them had a clinically significant anxiety disorder. However, they could not find significant correlations between anxiety and the severity of PD, duration of treatment, or levodopa dosage.

In the present study, we examined the presence of anxiety disorders in a consecutive group of patients with PD. Our main aim was to determine the presence of demographic, neurological, and cognitive correlates of anxiety in PD.

\section{PATIENTS AND METHODS}

\section{Patients}

A consecutive series of 40 patients with idiopathic PD who attended the neurology clinic at the Johns Hopkins Hospital were examined. Patients attending the clinic were seen for neurological evaluation or management of their disorder at regular follow-up visits. This clinic examined general neurology patients and was not specialized in treatment of patients with PD.

\section{Neurological examination}

The neurological examination was carried out by one of us (T.J.P) who was blind to the neuropsychiatric data. The evaluation consisted of a comprehensive neurological examination and a rating scale for symptoms of PD. The rating scale quantitated the presence and severity of tremor, rigidity and akinesia in both the left and right limbs (Fahn and Elton, 1987). Activities of daily living were quantified using the Northwestern Disability Scale (NWDS) (Canter et al., 1961). Based on clinical findings, the stage of illness was determined using the Hoehn and Yahr stages (Hoehn and Yahr, 1967).

\section{Psychiatric examination}

After giving informed consent, patients were administered a series of standardized quantitative measures of mood, cognitive function, and social connectedness. Examinations were administered in a private room between 
TABLE I. Demographic, neurological and psychiatric findings

\begin{tabular}{|c|c|c|c|c|}
\hline & $\begin{array}{l}\text { Anxiety and } \\
\text { depression }\end{array}$ & Anxiety & Depression & $\begin{array}{l}\text { No anxiety or } \\
\text { depression }\end{array}$ \\
\hline No of patients & 16 & 5 & 4 & 15 \\
\hline Age (years) & $65.3(10.0)$ & $63.2(9.9)$ & $68.2(9.2)$ & $70.0(9.3)$ \\
\hline Education (years) & $14.4(3.7)$ & $13.0(3.6)$ & $14.0(2.8)$ & $14.9(4.0)$ \\
\hline Sex (\% female) & 75 & 60 & 20 & 50 \\
\hline Duration (years) & $11.8(5.2)$ & $7.2(1.4)$ & $15.5(9.1)$ & $9.6(4.7)$ \\
\hline L-Dopa (mg) & 696 (439) & $710(283)$ & $825(507)$ & $663(384)$ \\
\hline Rigidity (score) & $3.2(1.6)$ & $2.6(1.5)$ & $3.7(1.7)$ & $3.2(1.6)$ \\
\hline Tremor (score) & $3.2(2.5)$ & $1.0(1.4)$ & $2.5(2.6)$ & $2.1(1.7)$ \\
\hline Akinesia (score) ${ }^{1}$ & $4.6(1.1)$ & $3.0(1.4)$ & $5.2(2.2)$ & $3.6(1.8)$ \\
\hline NWDS score ${ }^{2}$ & $14.3(8.6)$ & $5.4(2.6)$ & $15.5(12.4)$ & $8.4(3.9)$ \\
\hline PSE score ${ }^{3}$ & $15.9(4.5)$ & $5.2(2.0)$ & $11.0(1.0)$ & $3.0(3.2)$ \\
\hline HAM-D score ${ }^{4}$ & $11.9(2.9)$ & $7.0(1.4)$ & $7.0(2.5)$ & $3.2(3.2)$ \\
\hline HAM-A score 5 & $15.3(4.2)$ & $10.2(2.7)$ & $6.5(1.2)$ & $2.9(2.3)$ \\
\hline MMSE score ${ }^{6}$ & $26.3(5.2)$ & $27.6(1.5)$ & $23.0(6.9)$ & $28.8(1.3)$ \\
\hline STC score & $3.6(1.5)$ & $2.2(1.3)$ & $3.5(0.5)$ & $3.3(1.3)$ \\
\hline
\end{tabular}

${ }^{1} \mathrm{~F}(1,36)=7.23, p<0.01 ;{ }^{2} \mathrm{~F}(1,36)=4.0, p<0.05 ;{ }^{3} \mathrm{~F}(1,36)=6.71, p<0.05 ;{ }^{4} \mathrm{~F}(1.36)=15.1$, $p<0.001 ;{ }^{5} \mathrm{~F}(1,36)=41.8, p<0.0001 ;{ }^{6} \mathrm{~F}(1,36)=4.29, p<0.05$.

Means with S.D.s shown in parentheses.

$10.00 \mathrm{~h}$ and $14.00 \mathrm{~h}$ to minimize any possible effect of diurnal mood variation. The modified Present State Examination (PSE) (Wing et al., 1974), a semi-structured psychiatric interview that elicits symptoms related to depression and anxiety, was scored by the examiner. Based on the symptoms elicited using the structured interview (PSE), a psychiatric diagnosis of depression or anxiety disorders was made using DSM-III symptom criteria (APA, 1980). The Hamilton Rating Scale for Depression (HAM-D) (Hamilton, 1960) and the Hamilton Rating Scale for Anxiety (HAM-A) (Hamilton, 1959), measuring psychological and physiological symptoms of depression and anxiety, respectively, were completed by the interviewer. Cognitive impairment was quantified using the Mini-Mental State Examination (MMSE) (Folstein et al., 1975) (scores range from 0 to 30 with scores below 23 indicating significant cognitive impairment), and the number of social connections was quantified using the Social Ties Checklist (Starr et al., 1983) (scores range from 0 to 10 with higher scores indicating poorer social functioning).

\section{Statistical analysis}

Statistical analysis was carried out using means and standard deviations, one- and two-way analysis of variance (ANOVA), and $t$-tests. Correlations were calculated using a stepwise regression analysis. All $p$ values are two-tailed.

\section{RESULTS}

Twenty-one out of the 40 patients (52\%) met DSM-III criteria for GAD. In order to examine the correlates of anxiety, a stepwise regression analysis was carried out, using HAM-A scores as the dependent variable and age, MMSE scores, NWDS scores, duration of illness, and scores of tremor, rigidity and akinesia as independent variables. Depression scores (HAM-D) accounted for most of the variance $\left(\mathrm{R}^{2}=0.69, \mathrm{~F}=85.9, p<0.000001\right)$, and none of the remaining variables had enough power to enter the equation.

Sixteen of the 21 anxious patients (76\%) were also depressed [seven (44\%) had minor depression and nine $(56 \%)$ had major depression]. On the other hand, four of the 19 non-anxious PD patients ( $21 \%$ ) were depressed (all four with minor depression). Thus, there was a significant association between depression and anxiety in this group of PD patients $\left(\chi^{2}=14.76, \mathrm{df}=2, p<0.001\right)$. However, a significant association between anxiety and either major or minor depression was not statistically substantiated.

In order to further examine the association between demographic and neurological variables and psychopathology, patients were divided into anxious-depressed $(n=16)$, depression only $(n=4)$, anxiety only $(n=5)$, and no mood or anxiety disorder $(n=15)$ group.

\section{Demographic findings (Table I)}

A two-way ANOVA for duration of illness (factor 1: anxiety, factor 2: depression) showed a significant effect for depression (depressed patients had a significantly longer duration of illness) $[\mathrm{F}(1,36)=6.89, p<0.01]$. No significant between-group differences were found in the remaining demographic variables.

\section{Neurological findings (Table I)}

A two-way ANOVA for NWDS showed a significant effect for depression (depressed patients had significantly 
more severe deficits in activities of daily living) $[\mathrm{F}(1,36)=8.76, p<0.005]$. A three-way ANOVA for neurological signs [factor 1: neurological signs (tremor, rigidity, akinesia); factor 2: anxiety; factor 3: depression] showed a significant effect for depression $[F(3,24)=2.75$, $p<0.05$ ] (depressed patients had significantly more severe neurological signs than non-depressed patients). No significant between-group differences were found in the remaining neurological variables. None of the patients was on pergolide or selegiline.

\section{Psychiatric findings (Table I)}

A two-way ANOVA for MMSE scores showed a significant effect for depression (patients with depression showed significantly more severe cognitive deficits) $[\mathrm{F}(1,36)=5.28, p<0.02]$. No significant differences were found in Social Ties Checklist scores.

Several patients were on psychoactive medication. Two depressed patients were on antidepressants (imipramine 10 and $25 \mathrm{mg} /$ day, respectively), three patients with depression and anxiety were on imipramine (two on $25 \mathrm{mg} /$ day, and one on $100 \mathrm{mg} /$ day), and two anxious patients were on alprazolam ( $1 \mathrm{mg} /$ day $)$.

\section{DISCUSSION}

The present study examined patients with PD for GAD and showed several important findings. First, $40 \%$ of a consecutive and non-selected series of patients with PD met DSM-III criteria for GAD. Second, while anxiety was significantly associated with depression, some patients showed anxiety in the absence of depression. Third, while depression was significantly associated with long duration of illness and more severe cognitive and physical impairments, anxiety was not associated with greater impairment.

Before further discussion, several limitations of our study should be pointed out. First, we do not know the duration of anxiety in our PD patients, and only longitudinal studies will demonstrate the importance of anxiety as a pervasive psychiatric problem in PD. Second, since almost half of the patients with PD show depression, the diagnosis of anxiety may have been elevated by the overlap of anxiety and depressive symptoms (e.g. insomnia, decreased concentration, and restlessness may result from depression, but they are also diagnostic criteria for GAD). Finally, personality factors of PD patients may play an important role in the production of anxiety, and this issue will need further study.

The first important finding was the high frequency of anxiety ( $40 \%$ of a consecutive sample) in PD patients. The frequency is very similar to our previous finding of anxiety in stroke patients, as well as the frequencies of anxiety dis- orders in other reports of PD patients (Schiffer et al., 1988; Stein et al., 1990; Lauterbach and Duvoisin, 1991).

The question that now arises is the cause of anxiety disorder in patients with PD. One possibility is that anxiety may be a psychological response to the physical burden of the illness. However, in the study of Schiffer et al. (1988), patients with PD had significantly more severe anxiety than patients with multiple sclerosis and comparable physical impairments. Thus, other factors appear to be operative in the cause of anxiety disorder. Schiffer et al. suggested that anxiety in PD may result from either the pathological changes in $\mathrm{PD}$, or the use of dopaminergic agonists. While we found no support for the second hypothesis (i.e. there were no significant differences in duration of illness or levodopa dosage between groups with and without anxiety), the high frequency of anxiety in patients with PD may suggest that dysfunction in specific brain areas could account for this finding. Dysfunction of dopaminergic and noradrenergic pathways have been implicated in the production of anxiety (Roy-Byrne et al., 1986), and both the ventral tegmental area and the locus coeruleus (which give rise to mesolimbic dopaminergic and noradrenergic pathways, respectively) show significant neuronal loss in PD. However, the neuronal loss in these nuclei occurs at a different rate, and the locus coeruleus of non-demented PD patients shows a significantly greater reduction of dopamine compared with norepinephrine (Cash et al., 1987). Since dopamine decreases the firing rate of the locus coeruleus (Cederbaum and Aghajanian, 1977), Iruela et al. (1992) have recently speculated that the loss of dopamine inhibition of the locus coeruleus may underlie the presence of anxiety in PD. Since the imbalance between dopamine and norepinephrine concentration in the locus coeruleus decreases with the progression of PD and is not present in PD patients with dementia, Iruela et al. also speculated that the prevalence of anxiety should be lower in demented than in nondemented PD patients. However, we could not find a significant correlation between MMSE scores and anxiety scores, suggesting that the presence of anxiety is not linearly related to the severity of cognitive impairments. In any case, Iruela et al.'s hypothesis should be specifically tested in demented vs non-demented PD patients.

The second important finding was the significant association between anxiety and depression, suggesting that the presence of depression should be considered whenever a patient with PD complains of anxiety and vice versa. It should be noted, however, that anxiety may occur without depression, and some PD patients may show depression in the absence of anxiety. Taken together, these findings suggest that there may be at least two types of both major and minor depression in PD: an anxious depression, a non-anxious (perhaps apathetic or retarded) depression. Future studies may demonstrate whether non- 
anxious depressions are characterized by apathy or retardation and thus determine the validity of this theoretical construct.

In conclusion, our study demonstrated a high frequency of anxiety among a consecutive series of patients with PD, as well as a strong association between anxiety and depression. Whether this anxiety disorder, with or without depression, responds to the usual treatments for anxiety or depression remains to be determined.

\section{Acknowledgements}

This study was partially supported by a grant from the Raúl Carrea Institute of Neurological Research, the Sandoz Foundation, and the National Institutes of Mental Health grant \#R01 MH40355.

\section{REFERENCES}

American Psychiatric Association (1980) Diagnostic and Statistical Manual of Mental Disorders, 3rd edn. American Psychiatric Press, Washington, DC.

Canter GJ, de La Torre R and Mier M (1961) A method for evaluating disability in patients with Parkinson's disease. Journal of Nervous and Mental Disorders, 133, 143-147.

Cash R, Dennis T, L'Hereux R, Raisman R, Javoy-Agid F and Scatton B (1987) Parkinson's disease and dementia: norepinephrine and dopamine in locus coeruleus. Neurology, 37, 42-46.

Cederbaum JM and Aghajanian GK (1977) Catecholamine receptors on locus coeruleus neurons: pharmacological characterization. European Journal of Pharmacology, 44, 375-385.

Cummings JL (1992) Depression in Parkinson's disease: a review. American Journal of Psychiatry, 149, 443-454.

Fahn S and Elton E (1987) UPDRS Development Committee. Unified Parkinson's Disease Rating Scale. In: Recent Developments in Parkinson's Disease (Eds S Fahn, CD Marsden, M Goldstein and CD Calne). Macmillan, Florham Park, NJ.

Folstein MF, Folstein SE and McHugh PR (1975) Mini-Mental state: a practical method for grading the cognitive state of patients for the clinician. Journal of Psychiatry Research, 12, 189-198.

Hamilton MA (1959) The assessment of anxiety states by rating. British Journal of Medical Psychology, 32, 50-55.
Hamilton MA (1960) A rating scale for depression. Journal of Neurology, Neurosurgery and Psychiatry, 23, 56-62.

Hoehn MM and Yahr MD (1967) Parkinsonism: onset, progression and mortality. Neurology, 17, 427-442.

Iruela LM, Ibañez-Rojo V, Palanca I and Caballero L (1992) Anxiety disorders and Parkinson's disease. American Journal of Psychiatry, 149, 719-720.

Lauterbach EC and Duvoisin RC (1991) Anxiety disorders in familial parkinsonism. American Journal of Psychiatry, 148, 274.

Mayeux R, Stern Y, Rosen J and Leventhal J (1981) Depression, intellectual impairment, and Parkinson's disease. Neurology, 31, 645-650.

Roy-Byrne PP, Uhde TW, Sack DA, Linnoila M and Post RM (1986) Plasma HVA and anxiety in patients with panic disorder. Biological Psychiatry, 21, 849-853.

Schiffer RB, Kurlan R, Rubin A and Boer S (1988) Evidence for atypical depression in Parkinson's disease. American Journal of Psychiatry, 145, 1020-1022.

Starkstein SE, Preziosi TJ, Berthier ML, Bolduc PL, Mayberg HS and Robinson RG (1989) Depression and cognitive impairment in Parkinson's disease. Brain, 112, 1141-1153.

Starkstein SE, Bolduc PL, Mayberg HS, Preziosi TJ and Robinson RG (1990a) Cognitive impairments and depression in Parkinson's disease: a follow up study. Journal of Neurology, Neurosurgery, and Psychiatry, 53, 597-602.

Starkstein SE, Preziosi TJ, Bolduc PL and Robinson RG (1990b) Depression in Parkinson's disease. Journal of Nervous and Mental Disease, 178, 27-31.

Starkstein SE, Mayberg HS, Preziosi TJ and Robinson RG (1992) A prospective longitudinal study of depression, cognitive decline, and physical impairments in patients with Parkinson's disease. Journal of Neurology, Neurosurgery and Psychiatry, 55, 377-382.

Starr LB, Robinson RG and Price TR (1983) Reliability, validity and clinical utility of the Social Functioning Exam in the assessment of stroke patients. Experimental Aging Research, 9, 101-110.

Stein MB, Heuser IJ, Juncos JL and Uhde TW (1990) Anxiety disorders in patients with Parkinson's disease. American Journal of Psychiatry, 147, 217-220.

Wing JK, Cooper E and Sartorius N (1974) Measurements and Classification of Psychiatric Symptoms. Cambridge University Press, Cambridge.

(Received 18 July 1993; accepted 29 July 1993) 


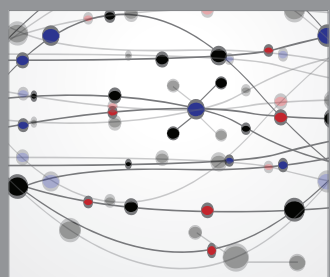

The Scientific World Journal
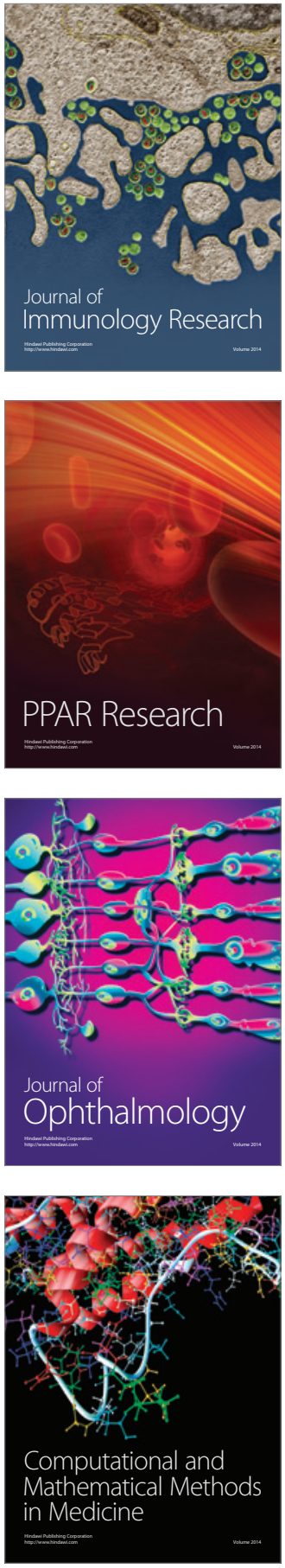

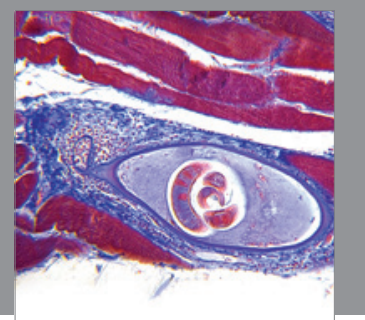

Gastroenterology

Research and Practice
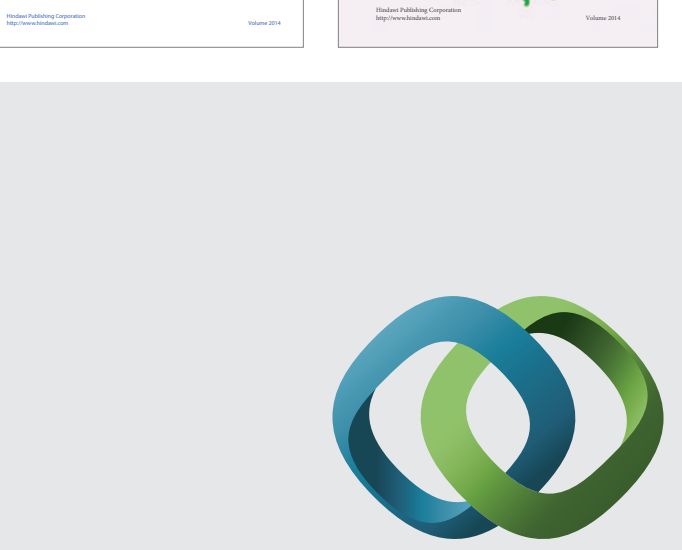

\section{Hindawi}

Submit your manuscripts at

http://www.hindawi.com
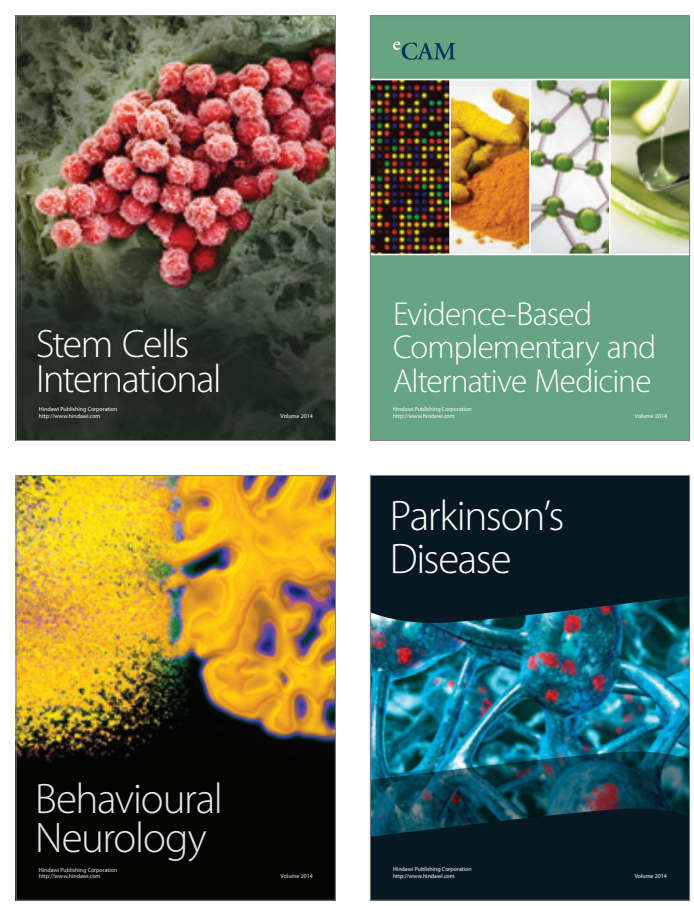

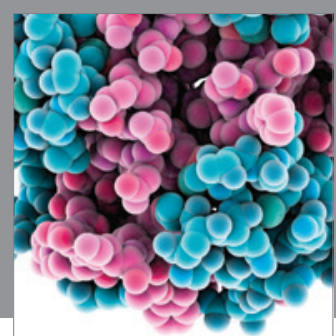

Journal of
Diabetes Research

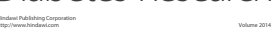

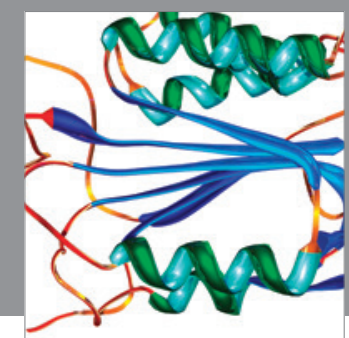

Disease Markers
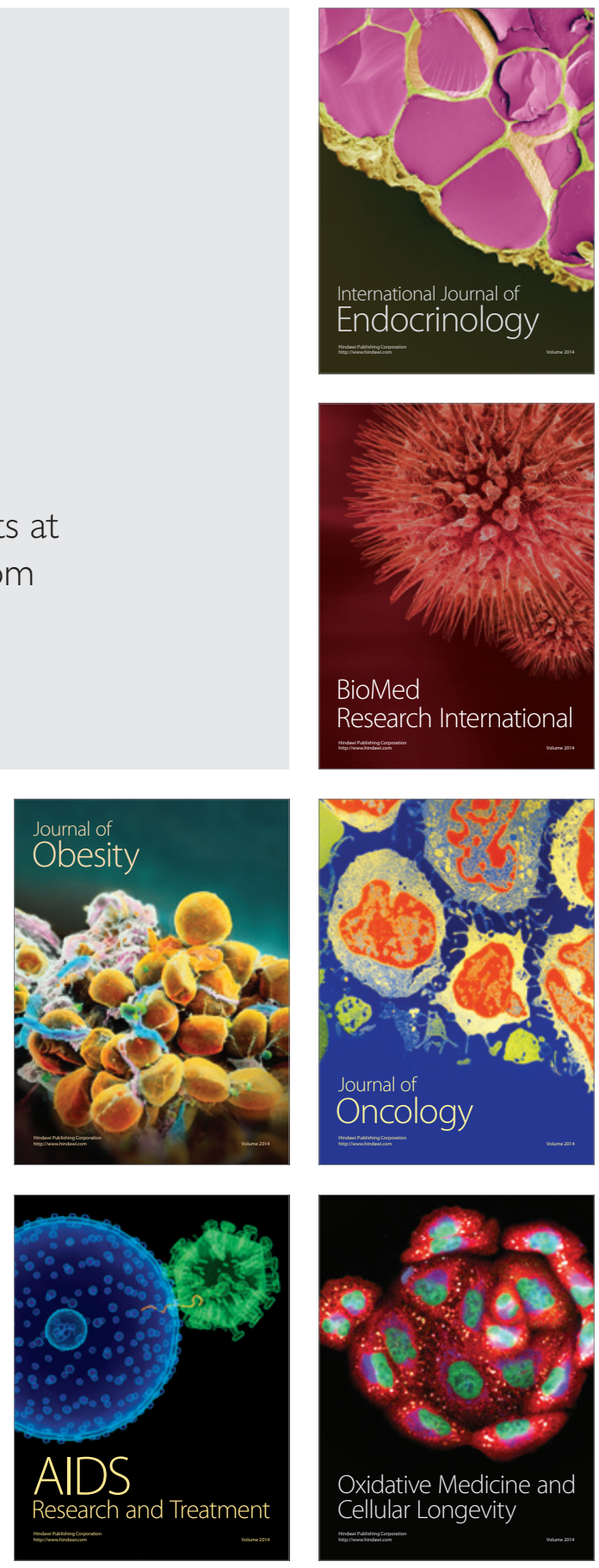